\title{
On a Technique to Relate EMF \& Rate of Change of Magnetic Flux
}

\author{
V. H. Doddamani ${ }^{1, *}$, K. H. Raveesha ${ }^{1}$, B. K. Prasad ${ }^{1}$, Ruchita Kumari ${ }^{2}$ \\ ${ }^{1}$ Department of Physics, CMR Institute of Technology, Bangalore, India \\ ${ }^{2}$ Department of Electronics Engineering, CMR Institute of Technology, Bangalore, India \\ *E-mail address: drvhdmani@gmail.com
}

\begin{abstract}
Faradays law of Induction is the principle of generating electricity in Hydro, Thermal, Wind and Nuclear power stations. In this paper we present a method to study the relationship between induced electromagnetic force (EMF) and the rate of change of Magnetic field and hence to verify this principle. This method involves the rotation of a magnet fixed to a rotor at different angular speeds and to find the EMF induced in the copper coil kept close to the rotor. The data collected is plotted in MATLAB. The results show that Induced EMF is directly proportional to the rate change of Magnetic flux with a correlation coefficient of 0.98 .
\end{abstract}

Keywords: Coil; Magnet; Rotor; MATLAB

\section{INTRODUCTION}

It is well known that Michael Faraday discovered the law of Induction. In his phenomenal paper 'Experimental Researches in Electricity', he stated that an EMF is induced in the coil when the magnetic field around the coil changes,( Phil. Trans. R. Soc. Lond. 1832 $122,125-162)$.He observed that the EMF induced is directly proportional to the rate of change of magnetic flux.

This law is the principle of Generators. A few articles are available in the literature on the methods of verifying this principle. We have conducted an experiment to study the relation between the EMF induced and the rate of change of magnetic field and hence to verify this law. It is proposed that this method could be set up easily in a laboratory enabling undergraduate science and engineering students to acquire

In the second section, the method is described along with the actual photographs of the experimental setup. In section three, the data obtained is presented. The graph of EMF induced versus rate of change of Magnetic field is shown. The MATLAB program utilized for this purpose is also provided in the appendix. The details of MATLAB program is provided in the appendix.

\section{METHOD}

Copper coil can be made by winding copper wire on a plastic frame. 100 grams of 46gauge copper wire was used to wind on a plastic frame. The number of turns was around 
20,000. 30 Gauge / 40 gauge copper wire also can also be used, but the size of the coil increases. The copper coil used in this experiment is shown in Fig 1.

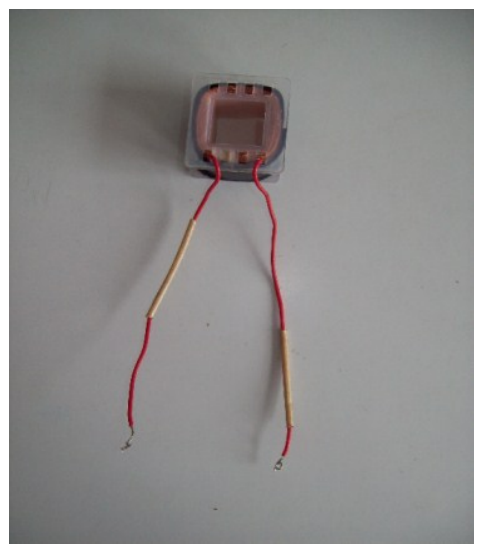

Fig. 1. Copper coil.

The magnet is glued strongly to the rotating disk. During rotation, due to centrifugal force, it tends to get ejected and may cause serious damage to the surrounding property. The magnet glued to the rotor is shown in the figure 2 .

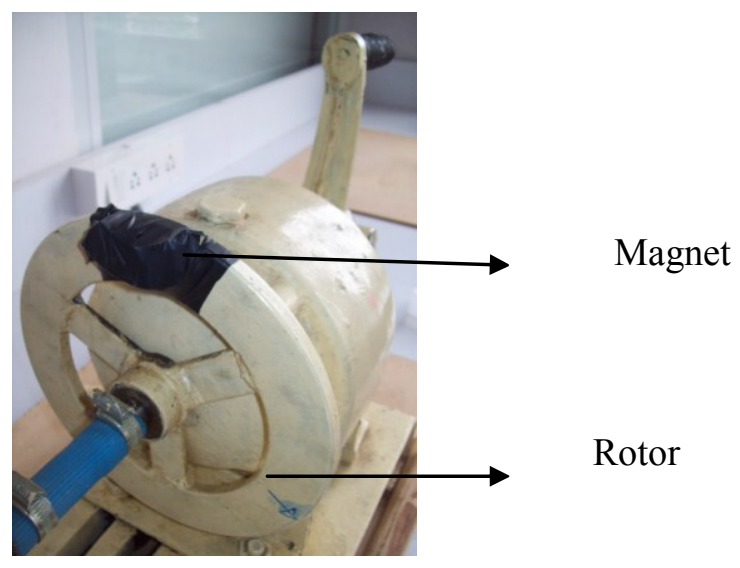

Fig. 2. Magnet fixed to the rotating disk.

The copper coil is kept closed to the rotor such that the magnet comes closer to the coil during its rotational motion. The closest separation distance in our set up was $5 \mathrm{~mm}$. The complete set up along with the voltmeter connected across the copper coil is shown in fig 3 . When the rotor is rotated, in each rotation the magnet arrives closer to the coil, an EMF is induced due to change in magnetic flux. As the rotational speed of the rotor is increased, the magnetic field around the coil changes at a faster rate. The corresponding induced EMF is measured. 


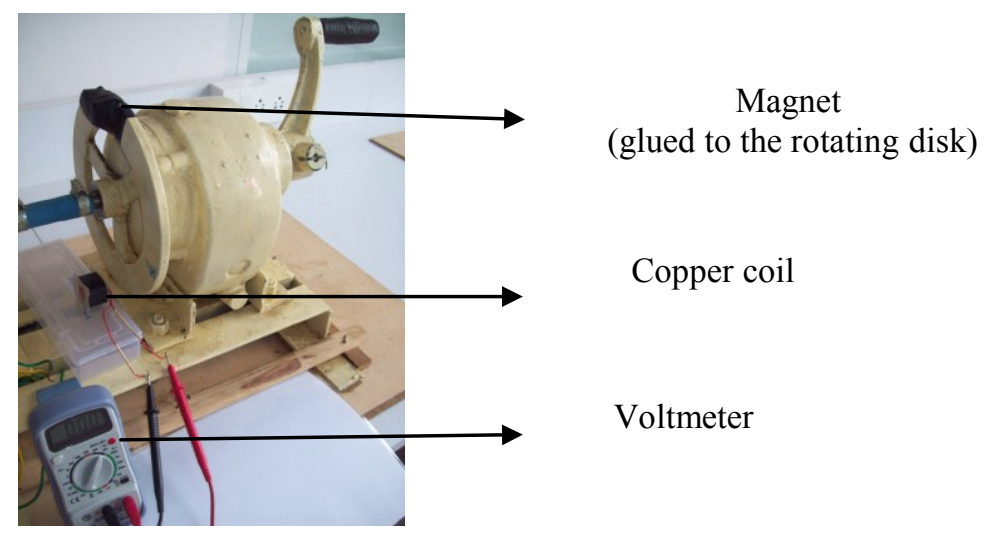

Fig. 3.

\section{DATA ANALYSIS}

The induced EMF at several rotational speeds is measured. It is assumed that the rotational speed is directly linked to rate of change of magnetic field. A graph of induced EMF versus rotational speed is plotted using MATLAB. The correlation coefficient between these two parameters is evaluated by least square fitting is 0.98 . The graph and the data is given in the following table.

\begin{tabular}{|c|c|}
\hline $\begin{array}{c}\text { Induced EMF } \\
(\mathrm{v})\end{array}$ & $\begin{array}{c}\text { Frequency of rotation } \\
\text { of the magnet } \\
(\mathrm{Hz})\end{array}$ \\
\hline 0 & 0 \\
\hline 0.5 & 5.52 \\
\hline 0.8 & 10.27 \\
\hline 1.5 & 13.86 \\
\hline 2.2 & 17.92 \\
\hline
\end{tabular}

It is known that induced EMF is proportional to rate of change of magnetic flux which is related to frequency of magnetic oscillating magnetic field.

Hence

$$
\begin{gathered}
\text { EMF } \mathrm{v} \alpha \text { frequency } \mathrm{f}^{\alpha} \\
\mathrm{v}=\mathrm{kf}^{\alpha} \\
\log \mathrm{v}=\log \mathrm{k}+\alpha \log \mathrm{f}
\end{gathered}
$$

This expression represents a straight line. So, in a plot of Induced EMF and frequency, the slope represents the power law index. The value of $\alpha$ in our study is close to 1 which 
confirms the linear relationship between Induced EMF and frequency of oscillating electric field.

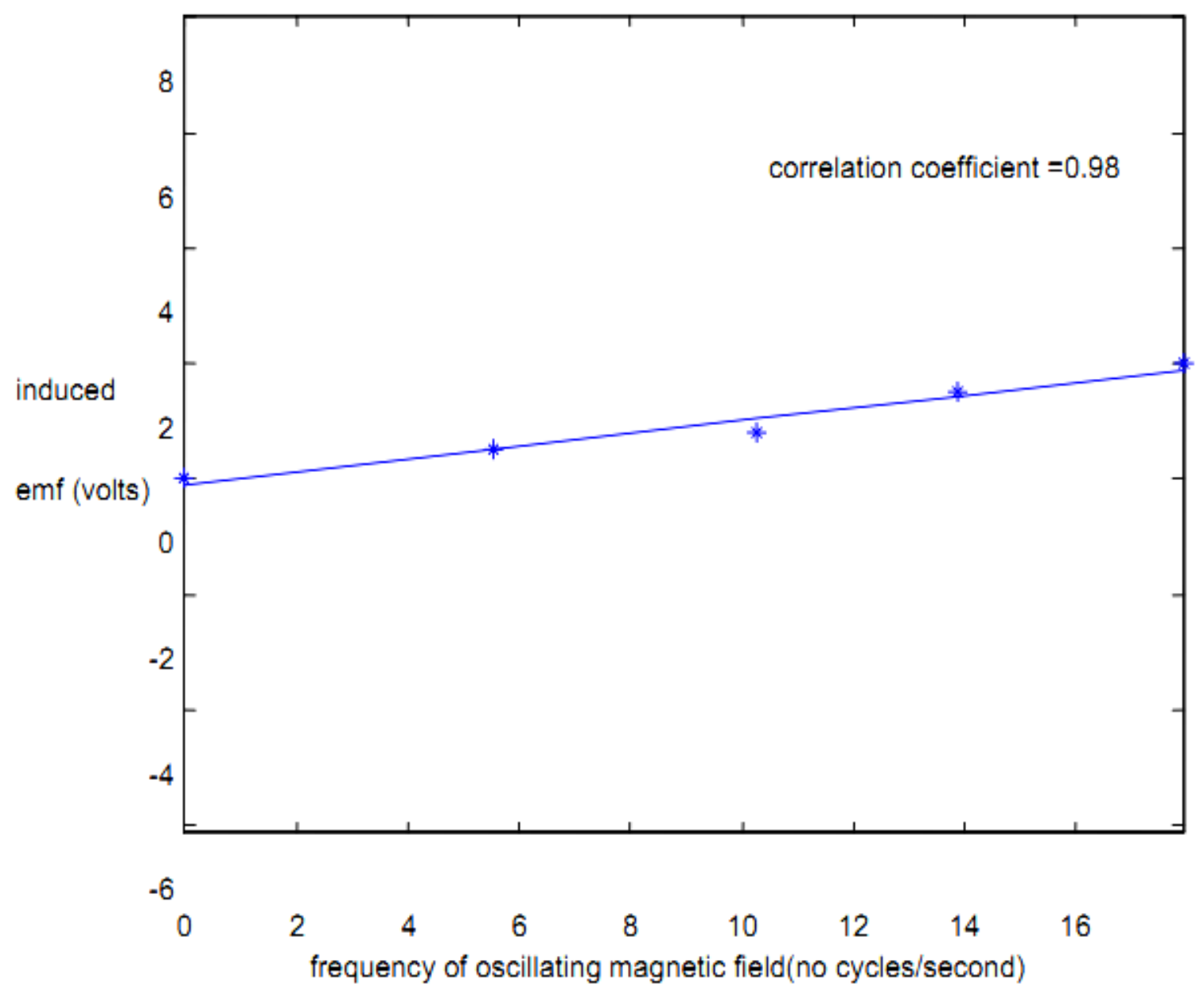

We opine that high correlation coefficient indicates that induced EMF is directly proportional to the rate of change of magnetic field (rotational speed of magnet).

Induced EMF $\alpha$ Rate of change of Magnetic field

\section{CONCLUSION}

The relation between the induced EMF in a coil due to the change in magnetic field linking with it is studied. The data indicates that the induced EMF is directly proportional to the rate of change in magnetic field.

\section{Appendix}

1.MATLAB program:

$\% \mathrm{x}$ is rotational speed; $\mathrm{y}$ is the induced EMF

$\mathrm{y}=\left[\begin{array}{lllll}0 & 0.5 & 0.8 & 1.5 & 2\end{array}\right]$ 


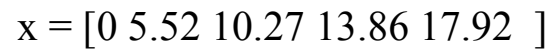

least

xlabel('frequency of oscillating magnetic field(no cycles/second)')

ylabel('induced emf (volts)')

axis equal

title(' Verification of Faradays Law Experiment')

end

\section{LEAST function}

$\%$ LEAST is a function to carry out least curve fitting i.e., to fit a straight line for a given set of points and hence to see how the two parameters are correlated \%

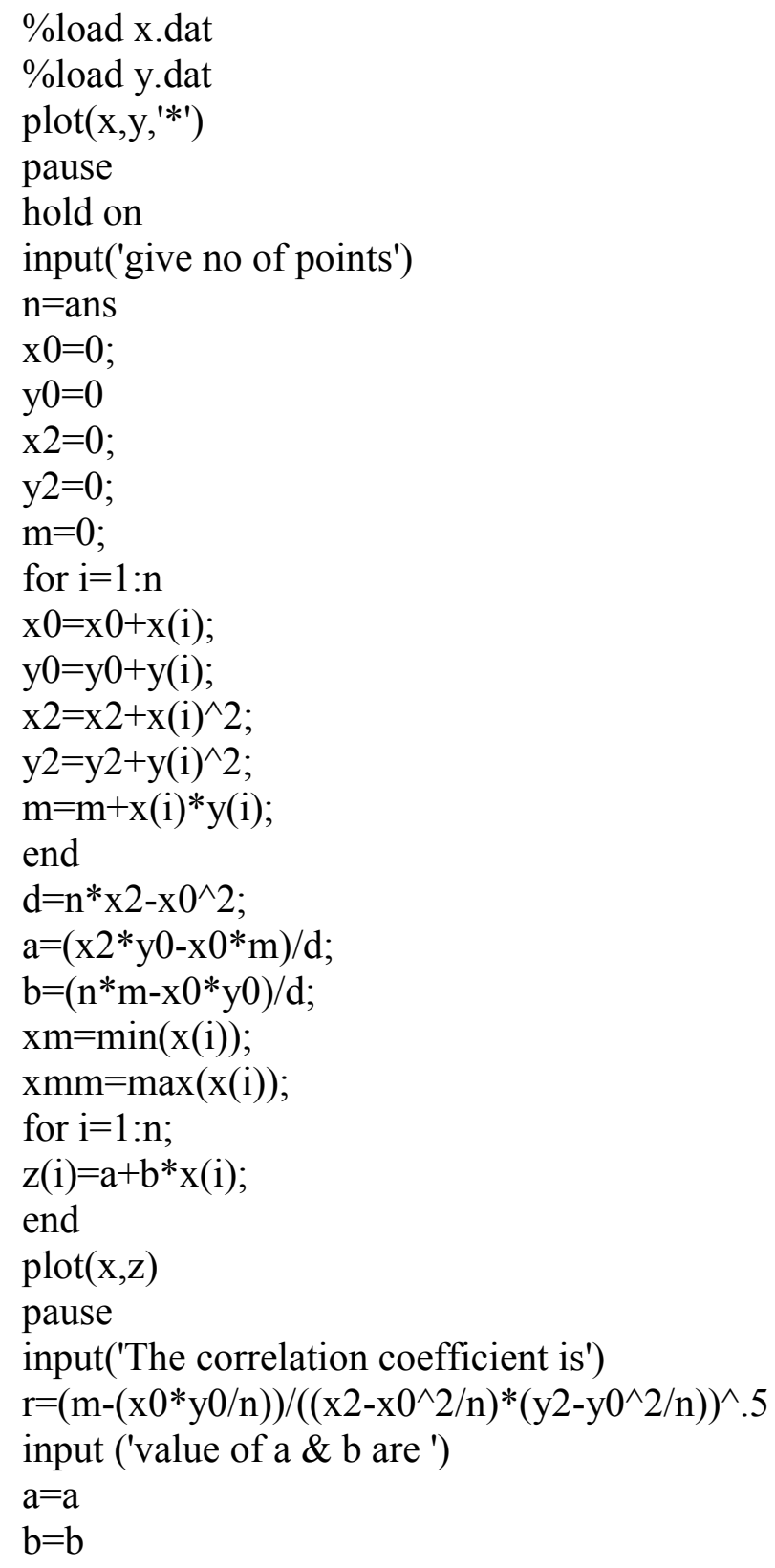




\section{Biography}

Dr Vijayakumar H Doddamani

Professor

Department of Physics

Bangalore University

Jnanabharathi campus

Off Mysore Road

Bangalore-56

Karnataka state

India

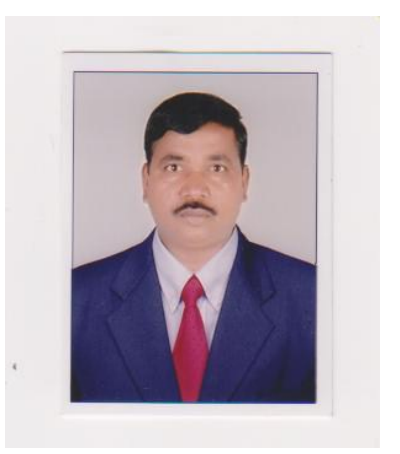

Dr V H Doddamani has been teaching post graduate and doctoral students from the past 20 years. His research areas include Active Galactic Nuclei, Radio astronomy, Plasma Physics etc. Under his guidance, several research scholars are working for $\mathrm{PhD}$. He has published research papers in reputed journals and has presented papers in national and international conferences. He is a member of several associations and academic committees contributing to the development of better teaching methodologies and curriculum. Currently, he is involved in designing Radio astronomy related experiments.

Raveesha KH

Faculty

Department of Physics

CMR Institute of Technology

IT Park Road

Kundalahalli

Bangalore-37

Karnataka state

India

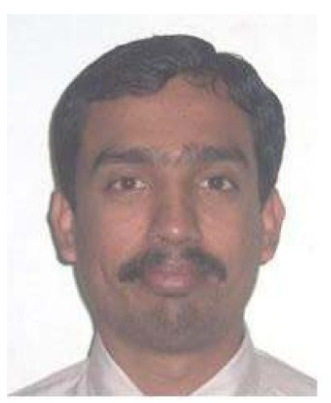

Raveesha KH has been teaching Physics for engineering students from the past 18 years. He is currently involved with designing lab experiments for undergraduate students. He is pursuing research in the field of Radio astronomy at Bangalore University at and Indian Institute of Astrophysics, Bangalore. His interests include designing Electromagnetics related experiments.

\section{References}

[1] Michael Faraday, Phil. Trans. R. Soc. Lond. 1832, 122, 125-162

[2] Oliver Heaviside, Electromagnetic Theory, London, Chelsea, 1897

[3] David J. Griffiths', Introduction Electrodynamics ( $3^{\text {rd }}$ Edition), New Delhi, Prentice Hall of India, 2004

[4] D. Cheng, Field and wave Electromagnetics ( $2^{\text {nd }}$ Edition), New Delhi, Pearson Education (India), 2002

[5] Nathan Ida, Engineering Electromagnetics( $2^{\text {nd }}$ Edition), New Delhi, Springer (India), 1998

[6] D. Chattopadyay et al., Electricity and Magnetism, Calcutta, Central, 2006,

[7] John D.Kraus, Electromagnetics ( $3^{\text {rd }}$ Edition), New York, McGraw-Hill, 2001

[8] J .D. Jackson, Classical Electrodynamics ( ${ }^{\text {rd }}$ Edition), New Delhi, Wiley India Edition, 2000 
[9] Feynman, Lectures on Physics, New Delhi, Narosa Publishing House, 1999

[10] Guru \& Hiziroglu, Electromagnetic Field Theory Fundamentals, New Delhi, Vikas publishing House, 2003

[11] Erik Hallen, Electromagnetic Theory, London, Chapman \& Hall, 1962 